\title{
BMJ Open Life or limb: an international qualitative study on decision making in sarcoma surgery during the COVID-19 pandemic
}

\author{
Samantha Bunzli, ${ }^{1}$ Penny O'Brien (1) , ${ }^{1}$ Will Aston, ${ }^{2}$ Miguel A Ayerza, ${ }^{3,4}$ \\ Lester Chan, ${ }^{5}$ Stephane Cherix, ${ }^{6}$ Jorge de las Heras, ${ }^{7,8}$ Davide Donati, ${ }^{9,10}$ \\ Uwale Eyesan, ${ }^{11}$ Nicola Fabbri, ${ }^{12,13}$ Michelle Ghert, ${ }^{14}$ Thomas Hilton, ${ }^{15,16}$ \\ Oluwaseyi Kayode Idowu, ${ }^{17,18}$ Jungo Imanishi, ${ }^{19}$ Ajay Puri, ${ }^{20,21}$ Peter Rose, ${ }^{22}$ \\ Dundar Sabah, ${ }^{23}$ Robert Turcotte, ${ }^{24,25}$ Kristy Weber, ${ }^{26}$ Michelle M Dowsey (D) , \\ Peter F M Choong ${ }^{1}$
}

To cite: Bunzli S, O'Brien P, Aston W, et al. Life or limb: an international qualitative study on decision making in sarcoma surgery during the COVID-19 pandemic. BMJ Open 2021;11:e047175. doi:10.1136/ bmjopen-2020-047175

- Prepublication history and additional supplemental material for this paper are available online. To view these files, please visit the journal online (http://dx.doi.org/10.1136/ bmjopen-2020-047175)

Received 20 November 2020 Accepted 18 August 2021

\section{Check for updates}

(c) Author(s) (or their employer(s)) 2021. Re-use permitted under CC BY-NC. No commercial re-use. See rights and permissions. Published by BMJ.

For numbered affiliations see end of article.

Correspondence to Dr Samantha Bunzli; samantha.bunzli@unimelb. edu.au

\section{ABSTRACT}

Objectives The COVID-19 pandemic is unprecedented as a global crisis over the last century. How do specialist surgeons make decisions about patient care in these unprecedent times?

Design Between April and May 2020, we conducted an international qualitative study. Sarcoma surgeons from diverse global settings participated in 60 min interviews exploring surgical decision making during COVID-19. Interview data were analysed using an inductive thematic analysis approach.

Setting Participants represented public and private hospitals in 14 countries, in different phases of the first wave of the pandemic: Australia, Argentina, Canada, India, Italy, Japan, Nigeria, Singapore, Spain, South Africa, Switzerland, Turkey, UK and USA.

Participants From 22 invited sarcoma surgeons, 18 surgeons participated. Participants had an average of 19 years experience as a sarcoma surgeon.

Results $17 / 18$ participants described a decision they had made about patient care since the start of the pandemic that was unique to them, that is, without precedence. Common to 'unique' decisions about patient care was uncertainty about what was going on and what would happen in the future (theme 1: the context of uncertainty), the impact of the pandemic on resources or threat of the pandemic to overwhelm resources (theme 2: limited resources), perceived increased risk to self (theme 3: duty of care) and least-worst decision making, in which none of the options were perceived as ideal and participants settled on the least-worst option at that point in time (theme 4: least-worst decision making).

Conclusions In the context of rapidly changing standards of justice and beneficence in patient care, traditional decision-making frameworks may no longer apply. Based on the experiences of surgeons in this study, we describe a framework of least-worst decision making. This framework gives rise to actionable strategies that can support decision making in sarcoma and other specialised fields of surgery, both during the current crisis and beyond.

\section{Strengths and limitations of this study}

- While task forces have mobilised to establish recommendations for patient prioritisation during COVID-19, and survey studies have explored the impact of COVID-19 on patient care, our study is the first to describe how clinical leaders make decisions at this unprecedented time.

- We applied a robust qualitative research methodology to uncover themes that pervaded the thinking of sarcoma surgeons in their decision making during COVID-19 and the impact of their decision making on centre-based multidisciplinary care.

- We included teams from 18 diverse international sites, at various points in the first wave of the pandemic, to understand if there was commonality in response and how this information would help to inform future strategies for the inevitable second and subsequent waves.

- The use of 'snowball sampling' to recruit the participants who were also invited to join the authorship team raises the potential that social desirability forces influenced interview responses.

\section{INTRODUCTION}

In the last century, natural disasters have typically been regional (eg, Ebola, Severe Acute Respiratory Syndrome (SARS) and Middle East Respiratory Syndrome (MERS)). The global nature of the current COVID-19 crisis makes it unprecedented as a pandemic in our lifetimes. How can we make the best decisions for our patients in these unprecedented times is a question at the forefront of clinicians minds the world over.

In naturalistic decision-making environments, pattern recognition helps to select optimal courses of action and predict outcomes. ${ }^{1}$ Failure to recognise a pattern from previous experience or training, ${ }^{2}$ in unfamiliar circumstances such as during a 
once-in-a-life time pandemic, can dramatically accentuate the complexity of healthcare delivery for frontline clinicians. By identifying the variety of decisions that experts have to make during a crisis, we can gain insight into the skills needed and strategies employed to successfully work through critical problems. This knowledge has particular relevance during a major national or international crisis that significantly impairs the supply, delivery and use of resources that impacts patient care, such as international war, natural disaster or pandemic.

The aim of this study was to investigate how expert sarcoma surgeons make decisions about the care of their patients during the COVID-19 pandemic. Sarcoma care is highly time dependent, resource intensive and combines the multidisciplinary approaches of diagnostics, surgery, chemotherapy and radiotherapy. ${ }^{3}$ Left untreated, limbs are lost at best, and the disease is fatal at worst. ${ }^{4}$ Encompassing the three priority areas of timing of treatment, integrating adjuvant therapies and selecting the appropriate surgical procedure, sarcoma surgery is an ideal context to study decision making as the findings will be applicable to other areas where surgery is the pivotal option.

Adopting a qualitative approach, we explored two specific research questions: what decisions do sarcoma surgeons have to make along the patient journey during COVID-19 and what cues (ie, relevant items of information) and rules are associated with each decision? We aimed to develop a framework that specialist teams in other fields could learn from and apply in practice, both during this pandemic and in future crises.

\section{MATERIALS AND METHODS}

\section{Study design}

In this study, we used a qualitative approach to study the processes that occur beneath the visible surface when expert sarcoma surgeons make decisions about patient care, not only what difficult decisions expert surgeons were faced with, but more importantly, how they made these decisions. The qualitative approach underpinning the study was reflexive thematic analysis. ${ }^{5}$ This approach enabled the research team to coconstruct meaning from the participants' responses through their 'lens' as clinicians and researchers with backgrounds in social science, physiotherapy, orthopaedic nursing and sarcoma surgery and generate themes that could inform patient care in future crises. Data were collected through semistructured interviews to facilitate rich understanding of surgical decision making in each participants' unique context. Information on the study timeline is presented in online supplemental figures 1 and 2.

\section{Patient and public involvement \\ No patient involvement.}

\section{Study setting}

This was an international study involving 18 centres, representing each continent (see table 1).

\section{Participants}

We sought to include surgeons from each continent, practising in a range of locations at different points in the pandemic. This is consistent with best practice in qualitative research that seeks to capture a range of diverse voices, rather than an average or single 'representative' voice. We started with a convenience sample, drawing on links between the lead investigator and an international network of limb salvage surgeons. We then used snowball sampling ${ }^{6}$ to identify and recruit additional surgeons practising in diverse settings that were in different phases of the first wave the COVID-19 pandemic. To be eligible for the study, surgeons had to be: (1) specialist sarcoma surgeons and (2) willing to participate in a 1-hour interview over a video conferencing platform. Participating surgeons were invited to join the authorship team and contribute to the interpretation of data and writing of this manuscript. Twenty-two surgeons were invited to participate via an email invitation from the lead investigator. Email invitations also included a study protocol that identified the occupation and role of each of the interviewers (SB and PO). Of these, 18 consented to participate, two did not respond, one passed the study details on to a colleague working in the same country without providing a reason for declining and one surgeon declined to participate. Recruitment and data analysis were conducted in parallel and recruitment ceased when data saturation was reached (when no new concepts were emerging in subsequent interviews) and we reached out target for diversity.

\section{Data collection}

Data from the Johns Hopkins University Coronavirus Resource Centre, which included total confirmed COVID-19 positive cases and deaths in each country, were tracked in each participant's region and country from the inception of the study (4 April 2020) until the conclusion of the study (7 August 2020).Timing of participants' interviews were recorded on each of the corresponding COVID-19 graphs (see online supplemental material 1 and figures 3-18) to provide context to their responses in relation to the severity of the pandemic at the time of interview. Further context was provided by general information about the impact of COVID-19 on each surgeon's specific health service (number of COVID-19 cases in their institution), the participant's sex, years of experience as an orthopaedic oncology surgeon and number of oncology patients treated in the past year.

Individual interviews were conducted by teleconference between 24 April and 19 May 2020. Interviews lasted $60 \mathrm{~min}$ and were audio-recorded for transcription purposes. These were anonymised and kept confidential from other participants. The interviews and data analysis were conducted by SB, a female qualitative researcher and musculoskeletal clinician (physiotherapist), supported by PO'B, a female social scientist. Interviewers engaged in a researcher reflection at the conclusion of each interview and also recorded field notes. In the interviews, participants were asked to think of a patient that they 
Table 1 Snapshot of COVID-19 context at time of interviews

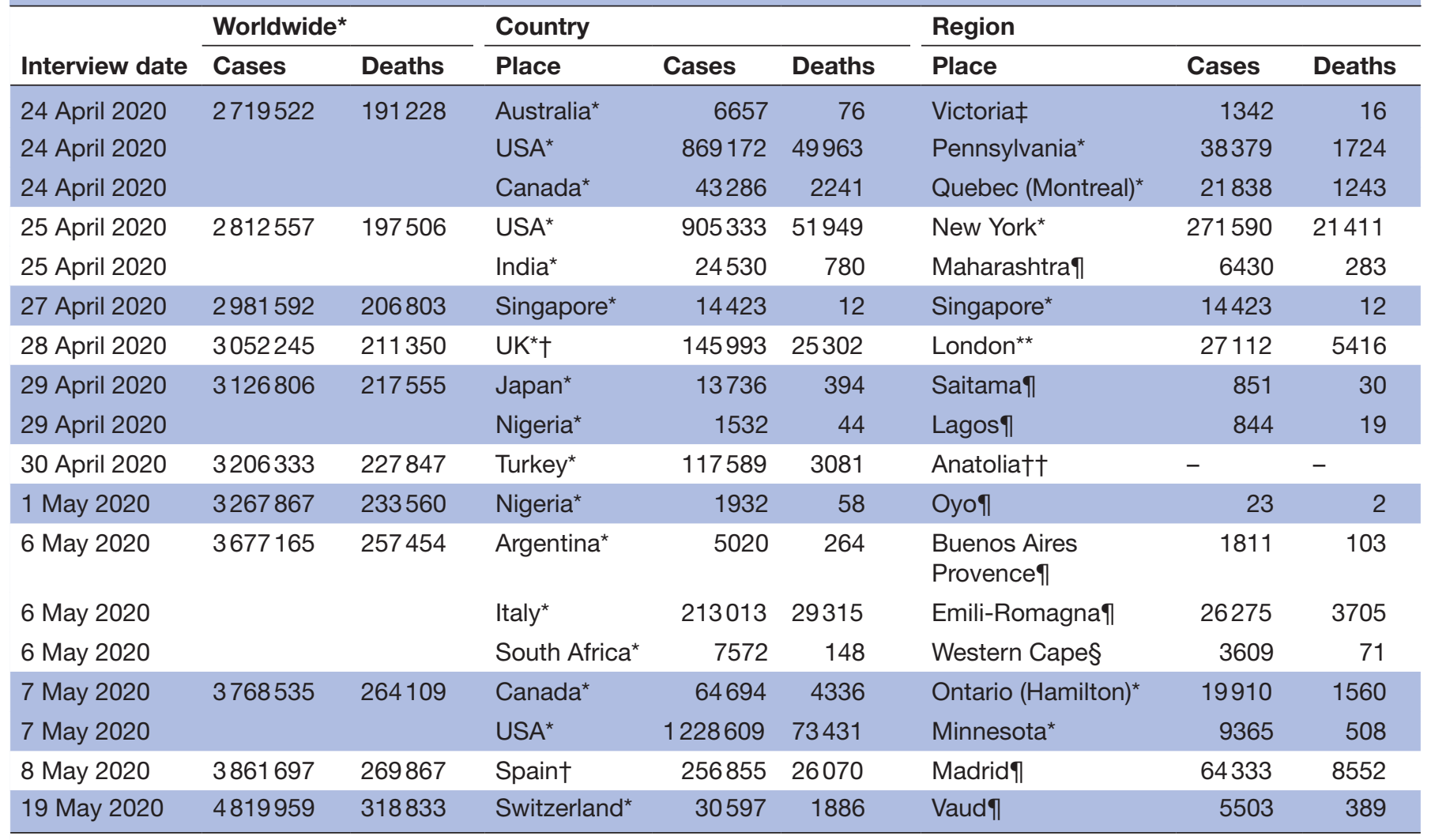

*https://coronavirus.jhu.edu/map.html

thttps://www.worldometers.info/coronavirus/\#countries

łhttps://www.covid19data.com.au/

$\S$ https://sacoronavirus.co.za

१https://en.wikipedia.org/wiki/Template:COVID-19_pandemic_data

${ }^{* *}$ https://coronavirus.data.gov.uk/details/deaths

††No data publicly available.

had treated during the pandemic and were prompted to think about the decisions they had to make throughout the patient's care (see online supplemental table 1).

\section{Data analysis}

Transcribed interview data were analysed using an inductive (data derived) thematic analysis. ${ }^{5}$ This began with 'open coding' in which concepts were identified in the interview data related to: (1) the decisions about patient care and (2) the cues and rules associated with these decisions. Two researchers (SB and PO'B) conducted open coding in duplicate, and a preliminary list of codes (coding framework) was compiled through consensus discussion. This coding framework continued to be refined through application to subsequent transcripts until it captured all relevant raw data. The refined framework was then applied to all 18 transcripts using the software NVivo (QSR international). Codes were grouped thematically, and preliminary themes were described following group discussion among the core research team in which alternative interpretations of the data were explored and debated. The core research team comprised of the two interviewer/analysts (SB and PO'B), the project lead and academic surgeon (PFMC), and an epidemiologist with content expertise in surgical decision making (MMD). Themes were then shared with the participating surgeons to check that they were an accurate interpretation of their experiences. Final themes were presented in narrative form and depicted in a figure (figure 1).

\section{RESULTS}

Eighteen sarcoma surgeons from 14 countries (table 1) were interviewed between April 2020 and May 2020. Participants had on average 19 years experience as a sarcoma surgeon. Ten participants had a mixed public and private caseload $(n=10)$, with eight operating only in public institutions (table 2).

At the time of the interviews, centres in Argentina, Canada, India, Japan, Nigeria, South Africa, Singapore, Turkey, the UK and the USA were on the ascending part of the first wave of the pandemic. Centres in Australia, Italy, Spain and Switzerland were reaching or on the plateau of the first wave (see table 1). 


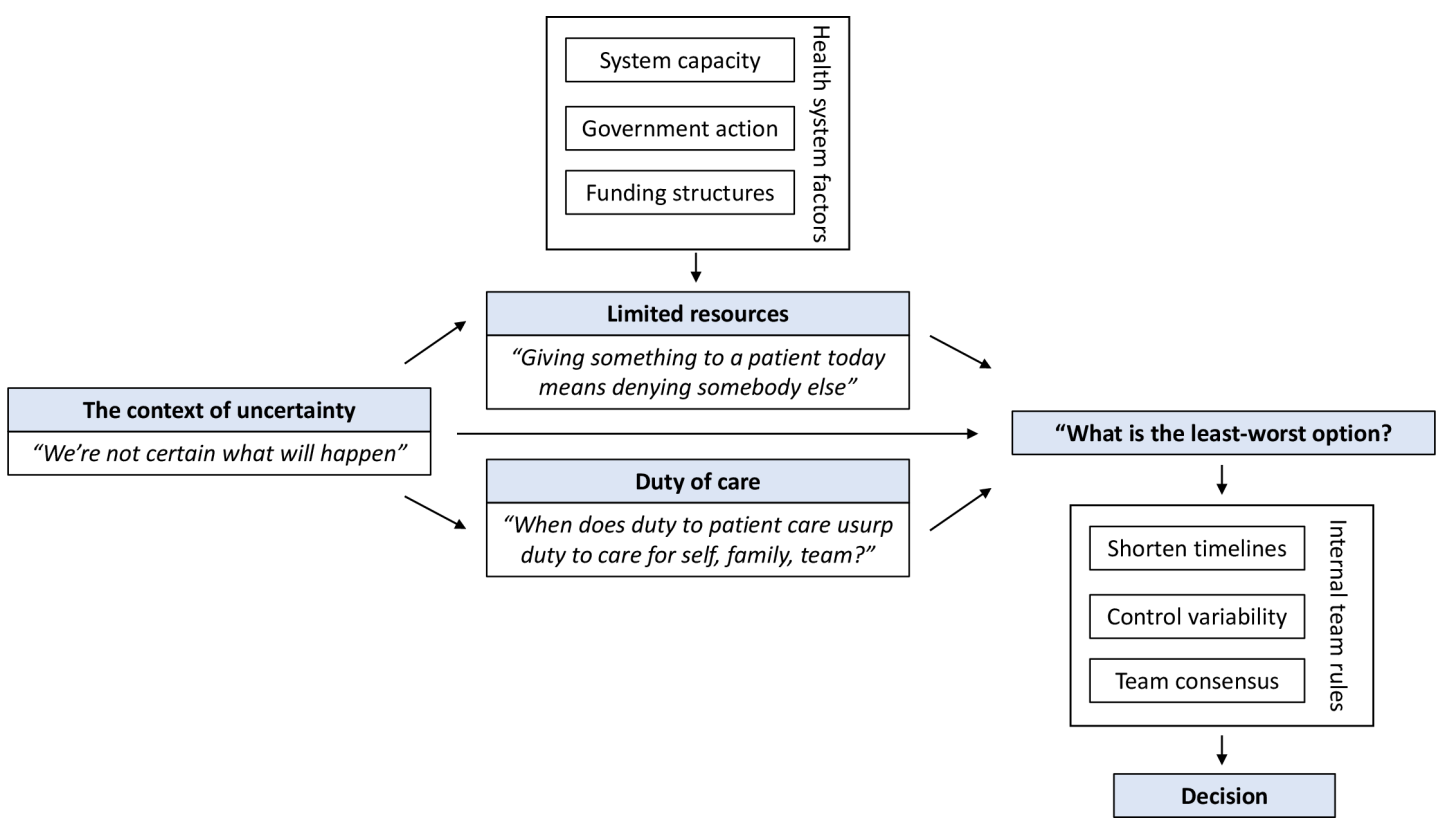

Figure 1 Framework of least-worst decision making in orthopaedic oncology care during COVID-19.

In total, 17 of the 18 participants described a decision they had had to make about patient care during the pandemic, which was unique to them; that is, they perceived there was no evidence or previous experience to draw on in making the decision (see box 1). The single participant who did not describe a unique decision

\begin{tabular}{|c|c|}
\hline Characteristic & Percentage participants (\%) \\
\hline \multicolumn{2}{|c|}{ Experience (years) } \\
\hline$<10$ & 22.2 \\
\hline $11-20$ & 33.3 \\
\hline $21-30$ & 38.9 \\
\hline$>30$ & 5.6 \\
\hline \multicolumn{2}{|c|}{ Time at institution (years) } \\
\hline$<10$ & 38.9 \\
\hline $11-20$ & 33.3 \\
\hline $21-30$ & 11.1 \\
\hline$>30$ & 16.7 \\
\hline \multicolumn{2}{|c|}{ Department surgeries* } \\
\hline$<250$ & 50.0 \\
\hline $250-500$ & 33.3 \\
\hline $501-1000$ & 11.1 \\
\hline$>1000$ & 5.6 \\
\hline
\end{tabular}

Public/private/mixed patient load

\begin{tabular}{lr} 
Public & 44.4 \\
Private & 0.0 \\
Mixed & 55.6 \\
\hline
\end{tabular}

*Number of orthopaedic oncology surgeries team performs per year (pre-COVID-19). (participant 06) had been redeployed to non-oncology clinical areas since the start of the pandemic. Therefore, the qualitative themes presented further relate to the 17 participants who reported unique decisions related to sarcoma surgery during the pandemic. Despite being in different phases of the pandemic, the key themes identified were common to the experiences of all 17 participants. Instances where diverse experiences occurred are reported within the description of each theme below.

Common to 'unique' decisions about patient care was the context of uncertainty (theme 1), limited resources (theme 2), duty of care (theme 3) and least-worst decision making (theme 4). These themes are described further, supported by quotes indexed by the participant number. Further contextual information for each participant is presented as additional quotes (see online supplemental table 2).

\section{The context of uncertainty}

The dynamic nature of the pandemic made it challenging for the participants to determine 'what is going on' and 'what will happen if...'. Without these key 'puzzle pieces', decision making was characterised by sentiments of uncertainty.

Participants emphasised the almost daily changes. Many, particularly those on the ascending part of the first wave, went to work each day not 'knowing what to expect': 'What has changed now is that on a daily basis I don't know what to expect' (participant 09). For those in leadership, the 'constantly changing' situation made it difficult to implement protocols and processes for their teams: 'You set in a protocol, you set in a system, that needs to be changed a week later just because the situation has changed' (participant 05).

Ambiguity about 'what was going on' made it challenging for participants to model outcomes of any course 


\section{Box 1 Examples of 'unique' decisions}

Participant 01: a decision is made to condense the course of radiotherapy into a shorter timeframe, accepting a reduction in the total dose and potential for greater side effects so that a patient can get to surgery sooner without becoming infected or the surgical team becoming infected.

Participant 02: a decision is taken to delay major surgery for a patient requiring an amputation that would take a lot of time and hospital resources and instead give additional chemotherapy to 'get further out of the pandemic' despite the risk that the tumour does not respond and potentially spread to the patient's lungs.

Participant 03: a decision is made to condense the course of radiotherapy as the radiation oncology staff have been told to reduce their case load, but this means that the surgeon has to find a spot for surgery a month earlier than anticipated, and it is possible the hospital will not have capacity by then.

Participant 04: a decision is made to give another round of chemotherapy to a patient who tests positive to COVID-19 just before surgery even though the patient is severely immunocompromised because a positive patient may struggle postoperatively and the theatre team would be at risk of exposure during surgery.

Participant 05: a decision is taken to defer surgery for large tumours that involve resource-intensive plastic surgery for a period of 8-10 weeks in the hope that the pandemic situation improves.

Participant 07: a decision is made to perform local flap procedures instead of large free flap procedures that require specialist services from a COVID-19 'hot-spot' hospital, as patients will be able to undergo chemotherapy for a couple of months until there is more capacity, even though local flaps may break down and need salvaging later.

Participant 08: a surgeon who is also a medical oncologist decides to act conservatively when treating patients with chemotherapy to reduce patients' vulnerability to infection.

Participant 09: a decision is made to operate on a patient with sarcoma, but the anaesthesiologists and scrub nurses are scared of doing anything that is not an emergency. So the hospital authorities intervene to help convince staff that this was an emergency case and without surgery the patient had a poor chance of survival.

Participant 10: a decision is made not to give preoperative radiotherapy, but instead to operate first and then give postoperative radiotherapy to reduce the risk of contamination by having to come regularly into a 'pandemic hospital'.

Participant 11: a surgeon who trained as a medical oncologist but stopped administering chemotherapy many years ago because of poor patient outcomes decides to administer chemotherapy to a patient who cannot access alternative chemotherapy services because of travel restrictions.

Participant 12: a surgeon in the 'at risk' category for COVID-19 who receives pressure from their own family to stop operating decides to operate on a young child whose COVID-19 status is unknown because the patient's family would not consent to a junior surgeon performing the surgery.

Participant 13: a decision is made to delay surgery and continue chemotherapy for patients with sarcoma.

Participant 14: a decision is made to put a patient with a large fracture requiring amputation in balanced traction and treat with chemotherapy when their surgery is cancelled because theatre staff went home to isolate. However, the oncology ward will not have a patient in traction, and chemotherapy cannot be given on the orthopaedic ward.

Continued

\section{Box 1 Continued}

Participant 15: a decision is made to bring surgery forward rather than wait for skin to heal after radiation because the hospital was starting to be closed down and the surgeon worried the patient would be 'shut out'. Participant 16: a decision is made to give radiation to a young child following a disfiguring amputation with a poor prognosis because 'we have gone this far, we don't want to stop'. However, the hospital will not allow both the child's parents to be present at the same time so the parents resort to lying to get past the 'guardians at the door'. The surgeon, charged with protecting others in the hospital, must tell the parents to stop this.

Participant 17: a decision is made that surgeries cannot go ahead because there are no more places available in the intensive care unit.

Participant 18: a decision is taken to operate first rather than give radiotherapy for a painful, growing tumour because of difficulties organising referral to radiotherapy in the early phases of the pandemic.

of action. The majority recounted the difficulty of planning a timeline for adjunct therapy and surgery when it was unclear what impact COVID-19 would have on hospital capacity and resources over time. In the following extract, a participant recounts how their hospital began to shut down in the early phases of the pandemic in preparation for the 'COVID-19 flood'. The participant refers to the 'radiation window' as the ideal time to schedule a strategy of preoperative radiotherapy and surgery but expresses concern that by the time this window is reached, hospital resources may no longer be available for non-COVID-19 patients: 'They were starting to close down the hospital and I was worried they were going to shut him out and delay his surgery beyond the radiation window, which would be really tragic' (participant 015). Several participants also recounted unforeseen disruptions that occurred during the surgical window that prompted life-saving surgery to be cancelled. These included travel restrictions, self-isolation of theatre staff and patients contracting COVID-19 just prior to surgery. The inability to predict when life-saving surgery would be able to go ahead made it difficult for participants to decide if and how to adjust the treatment plan. In the case of contracting COVID-19 just prior to surgery, a lack of evidence and clinical precedence meant participants were uncertain about how the virus would affect patients and impact on the surgical timeline, as recounted by this participant: 'She's currently asymptomatic, but we're uncertain if she'll never be symptomatic from the disease, or if she's about to blossom a very significant respiratory illness' (participant 016).

\section{Limited resources}

Making decisions was further challenged by the shift in the institutional model of care as the pandemic overwhelmed, or threatened to overwhelm, available resources. Shifting away from a model of shared decision making, institutions adopted a more utilitarian model of care in which resources had to be shared equitably with the greater 'collective good' in mind. Consequently, 
many participants were required to act as 'stewards of resources'.

Most participants reported an early establishment of patient triage guidelines that came from government health departments, recommending that all elective, nonemergency surgeries should be postponed to conserve resources. However, the participants explained that some sarcoma cases were more like emergency than elective cases, and being a rare cancer, sarcoma was usually not explicitly mentioned in the guidelines, leaving the decision to 'fall on the surgeon's shoulder': 'There've been some guidelines issued by the (government health department) regarding wait time for the different cancers. Sarcoma was not part of these guidelines. And this is the frustration - they always rely on your judgement for a final decision. So, the health department don't say don't do cancer surgery or do. They're just saying well, maybe things should be delayed, but it's for the surgeon to judge. So, basically, it always falls on your shoulder' (participant 03).

Except for those in settings most impacted by the pandemic who reported being unable to operate at the peak of the COVID-19 wave, the majority of participants were able to continue operating, with theatre access restricted to the most urgent surgical cases: 'We went from four theatres to one that was dedicated to orthopaedic oncology on our operating day as it were. And what that did was it made us be really clear about what would be on those lists, and we had to sit and discuss among ourselves what would the priorities $b e$ ?' (participant 01). In the following extract, a participant recounts how the need for resource stewardship meant that surgical procedures requiring 'disproportionate' hospital resources were automatically deferred to conserve resources for the impending influx of COVID-19 patients: 'So we haven't done major pelvic surgery because we don't want to have patients who are likely to require intensive care for a longer period of time. We also don't want to have patients who may require blood products beyond a certain known limit' (participant 05).

Deviating from previous best practice, particularly in order to benefit the 'collective good', could pose a threat to the therapeutic relationship between surgeons and their patients that had been built on a foundation of shared decision making. In the following extract, a participant in the peak of the first COVID-19 wave recounts their experience of 'informing' the parents of a young child that surgery cannot proceed due to limited resources: 'It's not because I don't want to operate or don't want to take good care of your child. But we are in the middle of a situation that's scary and we don't know enough. At the moment this is the best that we can do. This is genuinely what we think is best for your son, for our hospital, for the other patients. We have resources that are limited. We have to share those resources with other patients. And so if your son cannot be operated on now, we may devote this opportunity to somebody else' (participant 04). This extract captures the tension between acting in the best interests of the patient, while sharing resources justly with all patients requiring care. Participant 04 coped with this tension by rationalising the decision as "beyond their control': 'It makes me feel bad, but I think at personal level it sort of relieves me, because it's beyond my reach. It's not because I don't want to. It's not because I have done a bad operation and my margins should have been wider. It's not something that I'm in control of. I'm not in control of this. It's above me' (participant 04).

Other participants reframed the experience of 'resource stewardship' as 'balancing a more holistic picture', perceiving it as an opportunity to reconsider what is really necessary in patient care: 'In a pre-COVID era, there was no restraint. You could throw what you wanted at the patient. Today, giving something to the patient means denying somebody else something else, or you are adding further to the patient having to travel... And so you are balancing it in a more holistic picture - how much quality am I actually adding to the patient? Hopefully it will make some of us think about what is really important and how we should be looking at things' (participant 05). This participant practising in a 'rich country' where resources remained available despite the high prevalence of COVID-19, also saw the pandemic as an opportunity to rethink resource stewardship: 'We are $a$ rich country, so we have adapted activity to our means - maybe we follow-up our patients a bit too long. So we were able to postpone some without too much thought' (participant 18).

\section{Duty of care}

While committed to the ethical principle of beneficence (acting in the interests of others in need), fulfilling duties to patient care increased the participants' personal risk of being exposed to the virus and transmitting the virus to family members. Many older participants, who were themselves more vulnerable to the virus, experienced pressure from family members to stay away from active duty at the hospital. For these participants, deciding whether or not to operate on a patient involved a difficult trade-off between one's ethical duty to care for patients and one's ethical duty to care for oneself and one's family. This is captured in the following extract where a participant recounts their experience of caring for child in the early phases of the first COVID-19 wave when availability of virus testing was low: 'I received a lot of pressure from my family saying, "don't go to that surgery, you are going to be contaminated by the virus," and another pressure from the father's patient, saying to me, "No, doctor. I need you to be the surgeon." So it was a big deal (participant 012). Several participants drew on war-time metaphors to describe this 'ethical trade-off', finding meaning and purpose in being able to contribute to the 'war effort': 'So we are really waiting for the big wave and god knows if it's going to kill us or we're going to be swamped with COVID-19 patients. There is a mutual sentiment of, you know, we've got to be strong all together and go through all together... I think it has to do with the type of cancer that we treat... we swear to Hippocrates and this is just in line with that' (participant 04).

While the participants in this study all continued to provide patient care, several recounted stories of colleagues who had taken time away from patient care to look after their own mental health: 'Everyone handles the 
pressure differently. One of my junior staff members comes from a different city and was really struggling with their mental health. They needed to be with their family at this time, so they asked for a break' (participant 09)

\section{Least-worst decision making}

Participants faced with a lack of certainty about "what was going on', limited resources and a potential threat to self, engaged in least-worst decision making where none of the options were perceived as 'ideal', and the participants settled on the least-worst option at that point in time for each specific patient.

To decide on a least-worst option, participants in all phases of the COVID-19 wave applied common strategies, including: (1) shortening the timeline, that is, preferring short-term over longer-term treatment planning and goals; (2) controlling sources of variability which caused unpredictability, for example, choosing to give a COVID19-positive patient another round of chemotherapy rather than waiting to see when they would recover from virus to proceed with surgery; and (3) seeking consensus so that 'responsibility' for decision making was shared among members of the surgical oncology team.

In the following example, a participant considers two courses of action: either continue with a standard course of radiotherapy delivered over a 5 -week period or collapse the course of radiotherapy into a 2-week period. The participant explains that the first option would optimise the tumour for surgery but would involve more visits to hospital, thus increasing the risk of the patient being exposed to COVID-19. There was also a chance that the pandemic could overwhelm hospital capacity within the 5-week treatment period, resulting in the cancellation of surgery. In the second option, the 'finish line' (ie, surgery) would be reached sooner. However, a reduction in the total dose of radiotherapy could mean that the tumour is less optimised for surgery and the condensed dose of radiotherapy increased the chance that the patient would experience uncomfortable side effects. While neither option is 'ideal', the surgical oncology team and the patient decided that the worst option would be to 'not reach the finish line' and so the second option was selected as the 'least-worst': 'It's a bigger intensity, sometimes greater side effects but not as much of the (radiotherapy) dose is given over a shorter period of time. It means if they're lucky, they get through radiotherapy without becoming infected with COVID-19 or without the surgical team falling out from under them if they became infected. So it's really a balance of providing the best possible care, hoping to reach the finish line sooner than normal (participant 01).

In addition to changes to radiotherapy protocols, many participants described changes to chemotherapy protocols. For example, this participant recounts the risk balance of least-worst decision making when their patient tests positive to COVID-19 within 48 hours of surgery. Even though the patient is severely immunocompromised, the 'courageous' decision is made to postpone surgery and administer an additional round of chemotherapy while they wait for the patient to test negative: 'One patient with a high grade osteosarcoma of the femur and is undergoing preoperative chemotherapy... by the time that we were getting ready for surgery, the policy of testing the patient within 48 hours from the day of surgery was implemented and unfortunately, he tested COVID-19 positive. So this is a challenge on multiple level. Obviously it's a deviation from ideal treatment. Number two, it challenges at a cognitive level because this patient is severely immunocompromised. Which means that we take the courage essentially to give another round to chemotherapy to a patient possibly COVID-19 positive. This is a risk balance without precedent to make reference to. It's a combination of - gut feeling or courage or experience in trying to beat the cancer up as much as we can while at the same time caring about the pandemic' (participant 04).

Participants emphasised the importance of sharing least-worst decisions with the surgical oncology team and disruption to multidisciplinary tumour board meetings added to the experience of uncertainty in decision making for some participants. Institutions 'overrun' by the virus at the peak of the curve, cancelled multidisciplinary tumorboard meetings as all care focused on managing the flood of COVID-19 patients. For other institutions in the earlier phases of COVID-19, meetings were also cancelled with the rapid introduction of social distancing measures preventing in-person gatherings. Those with access to necessary infrastructure were able to continue with meetings over video conferencing platforms; however, these were often described as a 'shadow of their former selves'. 'I definitely missed the support of the team and that decision-making process. It gives you an added layer of comfort or reassurance that you are making the right decision. Even if they just agree with you, it's nice that people agree with you and I do miss those ones where it was less obvious. It's definitely been more difficult' (participant 014).

\section{DISCUSSION}

To our knowledge, this is the first qualitative study of surgical decision making during COVID-19. Under 'normal' circumstances (ie, prepandemic), decision making is driven by rational and recognition-primed choices. ${ }^{7}$ Having determined 'what is going on', decision makers consider multiple courses of action and select the action that offers a 'superior' outcome. ${ }^{8}$ In the abundance of resources, triage is based on the principle of need, with the sickest being the first to receive care. ${ }^{9}$ The ethical standard respects the autonomy of the patient and provider, taking into account their preferences through shared decision making. ${ }^{9}$ In the narrative accounts of decision making documented in this study, a paradigm shift was observed, most notably in the hospitals hardest hit by COVID-19. The factors that guided clinical decision making under 'normal' circumstances no longer applied, and a new decision making framework was revealed (see figure 1). Specific, actionable recommendations arising from this framework that may inform patient care in 
Table 3 Suggested strategies to support surgical decision making during COVID-19 and future crises

\begin{tabular}{|c|c|}
\hline Themes & Suggested strategies \\
\hline $\begin{array}{l}\text { The context of } \\
\text { uncertainty }\end{array}$ & $\begin{array}{l}\text { Establish strategy of clear and regular communication from institutional and clinical leaders. } \\
\text { Establish evidence-based practice guidelines for treatment rationalisation. } \\
\text { Maintain multidisciplinary consultations and discussion to ensure consensus decision making and } \\
\text { support. }\end{array}$ \\
\hline Limited resources & $\begin{array}{l}\text { Establish prioritisation system for personnel, consumable and treatment resources. } \\
\text { Establish split treatment teams to reduce vulnerability of cross-infection among clinicians and support } \\
\text { staff. } \\
\text { Establish 'designated survivor' status. } \\
\text { Ensure early communication and agreement between stakeholders within treatment teams of treatment } \\
\text { and diagnostic strategies. }\end{array}$ \\
\hline Duty of care & $\begin{array}{l}\text { Establish clear guidelines with regard to personal protective equipment. } \\
\text { Establish clear guidelines for institutional and personal guidelines for direct patient contact. } \\
\text { Establish prioritisation for shared (centre vs community) services, for example, investigations and } \\
\text { biopsy. } \\
\text { Minimise travel to and from treatment centres. } \\
\text { Broaden network of treatment facilities, for example, radiotherapy and chemotherapy. } \\
\text { Maintain multidisciplinary consultations to ensure optimal care. } \\
\text { Ensure patient support system exists. } \\
\text { Develop mechanisms to assess mental health of staff. } \\
\text { Provide clear institutional support for mental health needs of individuals and teams. }\end{array}$ \\
\hline $\begin{array}{l}\text { Least-worst } \\
\text { decision making }\end{array}$ & - Maintain multidisciplinary consultations to ensure decision support. \\
\hline
\end{tabular}

both sarcoma and other areas of life-saving surgery are presented in table 3 and discussed further.

\section{Least-worst decision making and the context of uncertainty}

Combat studies have revealed that decision makers lacking certainty about the outcome of a high-stakes decision identify 'workable' courses of action that match the situation and, committing to an unknown outcome, select the least-worst option at that point in time. ${ }^{10}$ Unlike military personnel, surgeons (particularly oncology surgeons) are rarely trained in 'disaster management'. 11 Despite this, the participants in our study applied recognised strategies in least-worst decision making, including shortening timelines, controlling sources of variability and seeking team consensus, $^{12}$ within the constraints of changing ethical standards of justice and beneficence principles in patient care. We recommend maintaining multidisciplinary consultations to ensure consensus decision making and support in the context of uncertainty (see table 3).

\section{Least-worst decision making and the context of limited resources}

In crisis medicine where resources are limited, the model of care shifts to benefit as many people as possible with available resources. ${ }^{9}$ In the place of shared decision making, a utilitarian model may emerge where decisions are made to be equitable for the greater 'collective' good. In this model, the patients most in need, who require the most resources, are the least likely to receive treatment. Guidelines have been published by the General Medical Council (GMC) stating that shared decision making is a fundamental component of good clinical practice. Therefore, surgeons should be supported to continue to adopt a model of shared decision making even in the most challenging of circumstances. While many participants in our study described situations in which limited resources impacted their clinical decision making, it is important to note that significant differences existed between health systems and supply capacities and how impacted these were by the pandemic. However, even in settings where resources were not overwhelmed during the ascending phase of the COVID-19 curve, restrictions were put in place in anticipation of the 'impending wave', and thus, participants in settings minimally impacted (eg, Australia) experienced reduced access to resources. While not on the frontline of the pandemic, surgeons have a responsibility to 'steward' limited resources to benefit the greatest number of patients. Medical associations have recognised that decision making under these conditions can be 'ethically challenging' and may conflict with doctor's 'moral intuitions'. ${ }^{13}$ Since data were collected for this study during the first phase of the pandemic, the GMC has published recommendations for doctors who face making challenging decisions about how to prioritise access to care within resources constraints. These recommendations include: taking account of local and national policies that set out criteria for accessing treatment; basing decisions on clinical need and likely effectiveness; and taking account of patients' wishes and expectations while also being transparent about decision-making processes and keeping a record of decisions made and reasons for them. ${ }^{14}$ Most importantly, and also reflected in our data, is that decision making in challenging situations should not rest with individual clinicians, rather support from colleagues and multidisciplinary teams 
should be sought. ${ }^{14} 15$ Standardising and documenting these ethical principles underlying triage during crisis is likely to help decision makers to manage distress. ${ }^{9}{ }^{16}$ As the participants in our study shifted to a model of 'collective justice', many sought to reduce distress by drawing on the strength of the patient-surgeon relationship, the consensus of a multidisciplinary team approach, applying strategies such as reframing (ie, 'balancing a more holistic picture') and avoiding rumination about decisions beyond their control. Based on the findings of our study, further recommendations to support decision making in the context of limited resources include early establishment of prioritisation systems (see table 3).

\section{Least-worst decision making and the duty of care}

While the 'duty of care' was a strong theme in our study, the crisis medicine literature reveals shifting boundaries around the principles of beneficence during a highly contagious pandemic. ${ }^{17} 18$ Clinicians have both an ethical obligation to care for their patients and themselves, raising the question: when does the duty to care for one's patients usurp the duty to care for oneself, and by extension, one's family? ${ }^{19}$ Such ethical obligations can be viewed as 'protected values', that is, values that are held 'sacred', more important than others and are not easily traded-off. ${ }^{20}$ Being forced to make decisions that violate protected values have the potential to cause 'moral injury'. ${ }^{21}$ While many of the participants in our study found meaning and purpose in contributing to 'the war effort', recognising the impact of any moral injury and distress, and how to support this, may play an important role in the 'return to normal' post-COVID-19. ${ }^{21}$ Recommendations to support surgeons in their 'duty of care' include implementing institutional processes to assess and support the mental health needs of individuals and teams (see table 3 ).

\section{Design considerations}

That the study participants were able to employ strategies to minimise distress may reflect our sampling strategy. It is possible that surgeons experiencing higher levels of distress were more likely to decline participation. Many participants described a range of reactions from members of their surgical teams, including elevated distress and disengagement from clinical work. It is possible that we only interviewed surgeons who were on the other end of this scale. The use of 'snowball sampling' ${ }^{6}$ to recruit the participants who were also invited to join the authorship team raises the potential that social desirability forces influenced interview responses. We attempted to minimise this by ensuring that the interviewers were unknown to the participants, by ensuring that interview responses were not shared with any other surgeon on the authorship team and the deidentified reporting of findings. With these assurances in place, participants shared their experiences honestly and openly, as reflected in the interview data (see online supplemental table 2).
Our findings resonate with other papers discussing triage decisions and resource allocation in oncology care and surgery during COVID-19. ${ }^{22-25}$ This suggests that our findings have applicability for surgeons practising in settings beyond those captured in our study. A key strength of our study is that we captured a global perspective of decision making in surgical oncology using a rigorous qualitative approach and found commonality in the responses despite surgical teams being at different stages of the first wave of COVID-19. Unlike survey studies exploring the impact of COVID-19 on surgical care (see, eg, refs 25-27), our qualitative approach enabled in-depth, novel insights into how surgeons make decisions during COVID-19 in 'real-time'. Participants had the opportunity to challenge/confirm these themes, and there was consensus agreement that the themes presented an accurate interpretation of their experiences. Finally, several guidelines have been published on patient prioritisation during COVID-19 since our interviews (see, eg, refs 22 23 28), and this may have influenced surgical decision making. Subsequent qualitative studies would be useful to capture how surgical decision-making changes over time.

Based on the findings of this unique, international qualitative study, we have identified strategies that may support decision making in sarcoma care both during the current crisis and beyond (see table 3 ). We suggest that while surgeons have evolved to be resilient, optimistic, self-sacrificing and just, profound anxieties can exist behind this face of professionalism. Establishing a robust, reliable and responsive network that can be used in any moment of peak or unexpected clinical demand would assist surgeons in times of need to leverage the support of their peers around the world.

\section{Author affiliations}

'Department of Surgery, St Vincent's Hospital Melbourne, The University of Melbourne, Melbourne, Victoria, Australia

${ }^{2}$ Royal National Orthopaedic Hospital London, London, UK

${ }^{3}$ Hospital Italiano de Buenos Aires, Buenos Aires, Argentina

${ }^{4}$ University of Buenos Aires, Buenos Aires, Argentina

${ }^{5}$ Tan Tock Seng Hospital, Singapore

${ }^{6}$ Service d'orthopédie et de traumatologie, Centre des sarcomes, Centre Hospitalier Universitaire Vaudois (CHUV), Lausanne, Switzerland

${ }^{7}$ Hospital La Paz, Madrid, Spain

${ }^{8}$ Universidad Autónoma de Madrid, Madrid, Spain

${ }^{9}$ IRCCS Istituto Ortopedico Rizzoli, Bologna, Italy

${ }^{10}$ Docente di Ortopedia e Traumatologia Università degli Studi di Bologna, Bologna, Italy

${ }^{11}$ Bowen University Teaching Hospital, Ogbomoso, Nigeria

${ }^{12}$ Department of Surgery, Orthopaedic Surgery, Memorial Sloan Kettering Cancer Center, New York City, New York, USA

${ }^{13}$ Weill College of Medicine, Cornell University, Ithaca, New York, USA

${ }^{14}$ Division of Orthopaedics, McMaster University, Hamilton, Ontario, Canada

${ }^{15}$ Groote Schuur Hospital, Cape Town, South Africa

${ }^{16}$ Red Cross Childrens Hospital, Cape Town, South Africa

${ }^{17}$ National Orthopaedic Hospital Lagos, Igbobi, Nigeria

${ }^{18}$ Bowen University, Lagos, Nigeria

${ }^{19}$ Saitama Medical University International Medical Center, Saitama, Japan

${ }^{20}$ Department of Orthopaedic Oncology, Tata Memorial Centre Department of Surgical Oncology, Mumbai, India

${ }^{21}$ Homi Bhabha National Institute, Mumbai, India

${ }^{22}$ Department of Orthopedic Surgery, Mayo Clinic, Rochester, Minnesota, USA 
${ }^{23}$ Department of Orthopedics and Traumatology, Ege University Faculty of Medicine, Izmir, Turkey

${ }^{24}$ McGill University Health Centre, Montréal, Québec, Canada

${ }^{25}$ Montreal General Hospital, Montréal, Québec, Canada

${ }^{26} \mathrm{Hospital}$ of the University of Pennsylvania, Philadelphia, Pennsylvania, USA

Contributors All authors attended regular meetings during the development and conduct of this study and/or received and provided feedback on meeting minutes. WA, MAA, LC, SC, JdIH, DD, UE, NF, MG, TH, OKI, JI, AP, PR, DS, RT, KW and PFMC provided data and contributed to the data analysis by reviewing and providing feedback on emerging interpretations. All authors reviewed the manuscript prior to submission. Specific contributions are: design: all; data collection: SB, PO'B and MMD; data analysis: $\mathrm{SB}, \mathrm{PO} B$ and MMD; data interpretation: all; manuscript writing: SB and PFMC; feedback on manuscript prior to submission: all. The first author, SB, is responsible for the overall content as guarantor.

Funding This work was supported by an Australian National Health and Medical Research Council Practitioner Fellowship held by PFMC (APP1154203). All authors were independent from funders, and all authors had full access to all of the data (including statistical reports and tables) in the study and can take responsibility for the integrity of the data and the accuracy of the data analysis.

Disclaimer The funder had no role in the study design; in the collection, analysis and interpretation of data; in the writing of the report; nor in the decision to submit the article for publication.

Competing interests None declared.

Patient consent for publication Not required.

Ethics approval Ethics approval was provided by St Vincent's Hospital Melbourne, Australia ID 63485 (LRR/066/20).

Provenance and peer review Not commissioned; externally peer reviewed.

Data availability statement Data are available upon reasonable request.

Supplemental material This content has been supplied by the author(s). It has not been vetted by BMJ Publishing Group Limited (BMJ) and may not have been peer-reviewed. Any opinions or recommendations discussed are solely those of the author(s) and are not endorsed by BMJ. BMJ disclaims all liability and responsibility arising from any reliance placed on the content. Where the content includes any translated material, BMJ does not warrant the accuracy and reliability of the translations (including but not limited to local regulations, clinical guidelines, terminology, drug names and drug dosages), and is not responsible for any error and/or omissions arising from translation and adaptation or otherwise.

Open access This is an open access article distributed in accordance with the Creative Commons Attribution Non Commercial (CC BY-NC 4.0) license, which permits others to distribute, remix, adapt, build upon this work non-commercially, and license their derivative works on different terms, provided the original work is properly cited, appropriate credit is given, any changes made indicated, and the use is non-commercial. See: http://creativecommons.org/licenses/by-nc/4.0/.

\section{ORCID iDs}

Penny 0'Brien http://orcid.org/0000-0002-0334-5288

Michelle M Dowsey http://orcid.org/0000-0002-9708-5308

\section{REFERENCES}

1 Klein G. Naturalistic decision making. Hum Factors 2008;50:456-60.

2 Islam R, Weir CR, Jones M, et al. Understanding complex clinical reasoning in infectious diseases for improving clinical decision support design. BMC Med Inform Decis Mak 2015;15:101.

3 Vodanovich DA, M Choong PF. Soft-tissue sarcomas. Indian J Orthop 2018:52:35-44.
4 Lawrenz JM, Featherall J, Curtis GL, et al. Time to treatment initiation and survival in adult localized high-grade bone sarcoma. Sarcoma 2020;2020:1-9.

5 Braun V, Clarke V. Reflecting on reflexive thematic analysis. Qual Res Sport Exerc Health 2019;11:589-97.

6 Moser A, Korstjens I. Series: practical guidance to qualitative research. Part 3: sampling, data collection and analysis. Eur J Gen Pract 2018;24:9-18.

7 Crebbin W, Beasley SW, Watters DAK. Clinical decision making: how surgeons do it. ANZ J Surg 2013;83:422-8.

8 Lipshitz R. Decision making in three modes. J Theory Soc Behav 1994;24:47-65.

9 Satkoske VB, Kappel DA, DeVita MA. Disaster ethics: shifting priorities in an unstable and dangerous environment. Crit Care Clin 2019;35:717-25.

10 Shortland N, Alison L, Thompson L, et al. Choice and consequence: a naturalistic analysis of least-worst decision-making in critical incidents. Mem Cognit 2020;48:1334-45.

11 Dennis AJ, Brandt M-M, Steinberg J, et al. Are general surgeons behind the curve when it comes to disaster preparedness training? A survey of general surgery and emergency medicine trainees in the United States by the eastern association for the surgery for trauma Committee on disaster preparedness. J Trauma Acute Care Surg 2012;73:612-7.

12 Lipshitz R, Strauss O. Coping with uncertainty: a naturalistic decision-making analysis. Organ Behav Hum Decis Process 1997;69:149-63.

13 British Medical Association. COVID-19-Ethical issues. A guidance note, 2021. Available: https://www.bma.org.uk/advice-and-support/ covid-19/ethics/covid-19-ethical-issues-when-demand-for-lifesaving-treatment-is-at-capacity

14 General Medical Council. How should doctors make decisions about prioritising access to treatment or care? London: GMC, 2021. https:// www.gmc-uk.org/ethical-guidance/ethical-hub/covid-19-questionsand-answers\#Decision-making-and-consent

15 General Medical Council. Good medical practice. London: General Medical Council London, 2013.

16 Ryus C, Baruch J. The duty of mind: ethical capacity in a time of crisis. Disaster Med Public Health Prep 2018;12:657-62.

17 Wong AH, Pacella-LaBarbara ML, Ray JM, et al. Healing the healer: protecting emergency health care workers' mental health during COVID-19. Ann Emerg Med 2020;76:379-84.

18 Holt GR. Making difficult ethical decisions in patient care during natural disasters and other mass casualty events. Otolaryngol Head Neck Surg 2008;139:181-6.

19 Grimaldi ME. Ethical decisions in times of disaster: choices healthcare workers must make. J Trauma Nurs 2007;14:163-4.

20 Baron J, Spranca M. Protected values. Organ Behav Hum Decis Process 1997;70:1-16.

21 Shortland N, McGarry P, Merizalde J. Moral medical decisionmaking: colliding sacred values in response to COVID-19 pandemic. Psychol Trauma 2020;12:S128-30.

22 Kutikov A, Weinberg DS, Edelman MJ, et al. A war on two fronts: cancer care in the time of COVID-19. Ann Intern Med 2020;172:756-8.

23 van de Haar J, Hoes LR, Coles CE, et al. Caring for patients with cancer in the COVID-19 era. Nat Med 2020;26:665-71.

24 Eng OS, Pawlik TM, Ejaz A. Cancer surgery during COVID-19: how we move forward. Ann Surg 2020;272:e94.

25 Hanna TP, Evans GA, Booth CM. Cancer, COVID-19 and the precautionary principle: prioritizing treatment during a global pandemic. Nat Rev Clin Oncol 2020;17:268-70.

26 Oba A, Stoop TF, Löhr M, et al. Global survey on pancreatic surgery during the COVID-19 pandemic. Ann Surg 2020;272:e87-93.

27 Benedetto U, Goodwin A, Kendall S, et al. A nationwide survey of UK cardiac surgeons' view on clinical decision making during the coronavirus disease 2019 (COVID-19) pandemic. J Thorac Cardiovasc Surg 2020;160:968-73.

28 Tzeng C-WD, Tran Cao HS, Roland CL, et al. Surgical decisionmaking and prioritization for cancer patients at the onset of the COVID-19 pandemic: a multidisciplinary approach. Surg Oncol 2020;34:182-5. 